\title{
BMJ Open Nurses' knowledge of peripherally inserted central catheter maintenance and its influencing factors in Hunan province, China: a cross-sectional survey
}

\author{
Binbin Xu (D , , ${ }^{1,2}$ Jinghui Zhang, ${ }^{1}$ Jianmei Hou, ${ }^{2}$ Mengdan Ma, ${ }^{2}$ Zhihong Gong, ${ }^{2}$ \\ Siyuan Tang ${ }^{2}$
}

To cite: Xu B, Zhang J, Hou J, et al. Nurses' knowledge of peripherally inserted central catheter maintenance and its influencing factors in Hunan province, China: a crosssectional survey. BMJ Open 2020;10:e033804. doi:10.1136/ bmjopen-2019-033804

- Prepublication history for this paper is available online. To view these files, please visit the journal online (http://dx.doi org/10.1136/bmjopen-2019033804).

Received 22 August 2019 Revised 18 April 2020 Accepted 05 May 2020
Check for updates

(C) Author(s) (or their employer(s)) 2020. Re-use permitted under CC BY-NC. No commercial re-use. See rights and permissions. Published by BMJ.

${ }^{1}$ Department of Respiratory Medicine, Xiangya Hospital Central South University, Changsha, Hunan, China

${ }^{2}$ Xiangya Nursing School, Central South University, Changsha, Hunan, China

Correspondence to

Mrs Jinghui Zhang;

zhangjh-1206@163.com

\section{ABSTRACT}

Objectives The present study aimed to assess the level of knowledge on peripherally inserted central catheter (PICC) maintenance among nurses in China and to analyse the related factors influencing this variable.

Design A cross-sectional survey.

Setting Ninety-one hospitals at three different levels in Hunan Province, China: county hospitals, municipal hospitals and provincial hospitals.

Participants A total of 4110 registered nurses engaged in clinical work related to intravenous infusion.

Primary and secondary outcome measures Nurses' knowledge of PICC maintenance was measured by the score of an anonymous, self-reported questionnaire. Results The mean score of PICC maintenance among 4110 nurses was $72.86 \pm 14.86$. $83.5 \%$ of the participants exhibited a score of 60 or above, and $34.1 \%$ of them exhibited a good grade with a score of 80 or above. The difference in the correct rate among different dimensions was statistically significant $(H=17.721, p<0.01)$. The generalised linear model indicated that the factors influencing the nurses' PICC maintenance knowledge included gender, age, professional title, work setting and previous history of PICC maintenance training.

Conclusions In conclusion, the knowledge of PICC maintenance was at a medium level among nurses in Hunan province, China. Multiple steps should be taken to improve the nurses' PICC maintenance knowledge, including disseminating PICC maintenance knowledge in multiple ways, such as courses, lectures, seminars and new media. Particular attention should be given to populations who responded poorly in this survey, and targeted education for nurses should be distributed based on their performance on specific dimensions, such as the replacement of dressing and needle-free connectors. In addition, the quality of the nurses' practical performance could be measured in the future.

\section{INTRODUCTION}

The peripherally inserted central catheter (PICC) is an intravenous device ${ }^{1}$ inserted into the central veins via the peripheral veins ${ }^{2-5}(\mathrm{eg}$, basilic vein, ${ }^{2}$ antecubital vein, ${ }^{2}$ cephalic vein, ${ }^{2}$ brachial vein ${ }^{3}$ and femoral vein),${ }^{45}$ which are commonly used for prolonged intravenous
Strengths and limitations of this study

- The present study is the first to compare nurses peripherally inserted central catheter (PICC) maintenance knowledge among hospitals at different levels in China.

- We designed the questionnaire based on an extensive review of literature and consultation with experts who are PICC specialist nurses and hold the position of head of the nursing department or head nurse.

- The sample size of the study was very large, which ensured the statistical power to support the conclusions.

- The cross-sectional design and convenience sampling method may cause non-response and selection biases and limit the reproducibility of the results.

The survey was based on self-reported data.

therapy, blood and nutrition administrations, and frequent blood sampling. ${ }^{4-10}$ It is regarded as a safe, efficient and cost-effective intravenous device due to long indwelling time, reduced vascular damage without repeated puncture, and convenient insertion and removal without the necessity for general anaesthesia or deep cutting and suturing of the wounds. ${ }^{2}{ }^{3-11}$ However, its complications cannot be ignored, including thrombosis, ${ }^{4}{ }^{12-14}$ catheter exit site infections, ${ }^{4}$ bloodstream infections, ${ }^{12} \quad 13 \quad 15$ accidental dislodgement, ${ }^{5} 11 \quad 12$ malposition, ${ }^{11}$ occlusion, ${ }^{45111216}$ leakage, ${ }^{12}$ breakage, ${ }^{5} 12$ phlebitis $^{12}$ and cardiac tamponade. ${ }^{15}$ A systematic review $^{17}$ indicated that approximately $30 \%$ of PICC failed before the completion of treatment because of complications, which would delay drug administration and blood sampling, increase the financial burden on patients and reduce their satisfaction. It was also shown that PICC-related complications can deplete patients' useable veins for future 
treatment and can cause long-term vessel obstruction. This could have a greater impact on cancer patients, since the delays to chemotherapy cycles might reduce treatment efficacy and could affect subsequent survival. Previous studies ${ }^{18-21}$ reported that appropriate PICC maintenance may offset the risk of such harms and maximise the safety of PICCs. The nurses' PICC maintenance knowledge and skills directly affect the quality of care, clinical outcomes and patient safety. ${ }^{82}$ Although gaps have been reported between the evidence of PICC maintenance and the knowledge and practice of nurses, ${ }^{38}$ little is known regarding nurses in Hunan province, China. Therefore, the purpose of the present cross-sectional study was to investigate the nurses' knowledge of PICC maintenance. We also aimed to characterise and identify some factors influencing nurses' knowledge, which may provide information on decision making and quality-improvement efforts associated with PICC.

\section{METHODS}

\section{Study setting}

To investigate the nurses' knowledge of PICG maintenance, we conducted a web-based cross-sectional survey of nurses in 91 hospitals at three different levels: county hospitals, municipal hospitals and provincial hospitals. County hospitals are hospitals located in counties that are under the control of county governments and provide comprehensive health services and medical education to residents in counties, villages and towns. They are generally grade II hospitals. Municipal hospitals are hospitals constructed and administered by the municipal governments. They are located in cities and provide comprehensive health services, emergency and critical medical services, and specialist health services to residents from the different counties. They also perform a wider role in medical education and conduct research on a regional basis. They are mainly grade III hospitals. However, municipal hospitals which are too small and do not contain sufficient beds (less than 500) are grade II hospitals. Provincial hospitals are hospitals under the jurisdiction of provincial governments. They are usually located in the provincial capital and play an important role in scientific research and teaching. They serve as medical hubs providing care to multiple regions. They are mainly grade III level A hospitals.

All included hospitals are members of the PICC maintenance service network in Hunan province, China. The PICC maintenance service network is a province-wide PICC maintenance alliance led by the intravenous infusion team of the Xiangya Hospital, Central South University. This network aims to allow patients in the intermission of treatment to select the nearest site to maintain their PICCs instead of returning to the original site where the PICC was placed. This ensures that PICC maintenance is more convenient and cost-effective. The Xiangya Hospital is a university-affiliated hospital with 3620 beds and a training base for PICC specialist nurses in Hunan
Province. The intravenous infusion team of the Xiangya Hospital began to construct the PICC maintenance network in 2015. To date, 103 maintenance sites have been involved. Each maintenance site has an intravenous infusion team that is responsible for intravenous nursing training (peripheral venous catheter training, central venous catheter training, PICC training and Implantable Venous Access Port training), quality control and consultation. Each intravenous infusion team includes a liaison who is responsible for communicating with the intravenous infusion team in other hospitals.

\section{Participants}

Participants were enrolled through a non-random, convenience sampling method. Initially, we contacted the liaisons in every PICC maintenance site and enquired whether they could aid us to carry out the survey. Subsequently, in sites where the liaisons were willing to aid us, registered nurses (RNs) who engaged in clinical work related to intravenous infusion were included. The inclusion criteria were as follows: RNs with more than 1 year of work experience; RNs who were willing to participate in the research after informed consent and RNs who can correctly understand the content of the questionnaire. The exclusion criteria were RNs who were receiving psychotropic substances due to mental or psychological illness and RNs who were absent from work due to illness, maternity leave or other reasons.

The sample size was calculated according to the following formula:

$$
n=\frac{z_{\alpha / 2}^{2} \sigma^{2}}{\delta^{2}}
$$

$\mathrm{Z}_{\alpha / 2}$ is the abscissa of the normal curve that cuts off an area $\alpha$ at the tails; $\sigma$ is the population standard derivation, which could be replaced by the sample $\mathrm{SD} ; \delta$ is the margin of error, the value of which is generally $10 \%-60 \%$ of the SD. In the present study, these factors were calculated as follows: $\alpha=0.05, Z_{\alpha / 2}=1.96, \sigma=14.602$ (determined by the presurvey), $\delta=1.4602(10 \% * \sigma)$. Therefore, the sample size was estimated to $n=384$. If non-response and invalid response subjects were considered, the sample size was increased by $20 \%$, corresponding to 461 .

\section{Survey instrument}

In the present study, the survey instrument was a selfdesigned questionnaire based on an extensive review of literature, ${ }^{81823-25}$ mainly including the Infusion Therapy Standards of Practice ${ }^{23}$ released by the Infusion Nursing Society and the Nursing Practice Standards for Intravenous Therapy ${ }^{24}$ issued by the National Health Commission of the people's Republic of China in 2013. Besides, five experts who were PICC specialist nurses and held the position of head of the nursing department or head nurse were consulted to assess the questionnaire's validity. A pretest was carried out to ensure that the questionnaire could be understood easily. The questionnaire included 50 items in total covering five dimensions of PICC maintenance knowledge, including PICC flushing and locking 


\begin{tabular}{|c|c|c|}
\hline Characteristic & Frequency & Percentage (\%) \\
\hline \multicolumn{3}{|l|}{ Gender } \\
\hline Male & 58 & 1.4 \\
\hline Female & 4052 & 98.6 \\
\hline \multicolumn{3}{|l|}{ Age, years } \\
\hline$<25$ & 1268 & 30.9 \\
\hline $25-34$ & 2120 & 51.6 \\
\hline $35-44$ & 597 & 14.5 \\
\hline$\geq 45$ & 125 & 3.0 \\
\hline \multicolumn{3}{|l|}{ Professional title } \\
\hline Nurse & 1554 & 37.8 \\
\hline Senior nurse & 1555 & 37.8 \\
\hline Supervisor nurse & 848 & 20.6 \\
\hline Cochief nurse & 153 & 3.7 \\
\hline \multicolumn{3}{|l|}{ Education level } \\
\hline $\begin{array}{l}\text { Technical secondary } \\
\text { school }\end{array}$ & 49 & 1.2 \\
\hline Junior college & 1906 & 46.4 \\
\hline Undergraduate & 2130 & 51.8 \\
\hline Postgraduate & 25 & 0.6 \\
\hline \multicolumn{3}{|l|}{ Work setting } \\
\hline County hospital & 1228 & 29.9 \\
\hline Municipal hospital & 1929 & 46.9 \\
\hline Provincial hospital & 953 & 23.2 \\
\hline \multicolumn{3}{|c|}{ Whether PICC maintenance training was obtained before? } \\
\hline No & 2277 & 55.4 \\
\hline Yes & 1833 & 44.6 \\
\hline
\end{tabular}

PICC, peripherally inserted central catheter.

(12 items), replacement of dressings and needle free connectors (8 items), complication management (19 items), health education (6 items) and catheter removal
(5 items). All items were single choice questions. The correct answer was assigned a point of 2, otherwise 0 was inserted, with a full score of 100 . The higher the score, the higher the PICC maintenance knowledge level. The Cronbach's alpha coefficient of this questionnaire was 0.873 , and the Content Validity Index was 0.915 , indicating that the questionnaire had good internal consistency reliability and validity.

In addition, we collected the data of potential factors influencing PICC maintenance knowledge by a selfdesigned demographic information questionnaire. These included gender, age, professional title, education level, work setting and previous history of PICC maintenance training.

\section{Implementation}

This survey was divided into the two following stages: The recruitment of PICC maintenance sites and the participants and the implementation of the investigation.

In June 2017, we recruited 91 PICC maintenance sites out of 103 eligible sites. After the recruitment of PICC maintenance sites, we provided unified training on our inclusion and exclusion criteria for these liaisons by telephone. The recruitment of the participants was implemented by these liaisons in July 2017. The specific steps used were as follows: initially, the liaisons explained the purpose of the survey to the head nurses in their facilities to obtain approval and support. Subsequently, the liaisons selected the potential participants according to the study inclusion and exclusion criteria with the help of the head nurses. Finally, the participants were included following their informed consent. All eligible nurses were informed that participation in this study was voluntary. They could withdraw from the study at any time for any reason, and the questionnaire would be answered anonymously based on their own knowledge and understanding of PICC maintenance. Moreover, they were assured that their information would only be used for research, their data would be kept confidential, and their scores of the questionnaire would not have any influence on their

Table 2 Score and distribution of PICC maintenance knowledge among the 4110 included nurses

\begin{tabular}{|c|c|c|c|c|c|}
\hline \multirow[b]{2}{*}{ Dimensions } & \multirow[b]{2}{*}{ Score $(\overline{\mathrm{x}} \pm \mathrm{s})$} & \multicolumn{4}{|c|}{ Grade of the total score* } \\
\hline & & Failed, $\mathbf{N}(\%)$ & Passed, N (\%) & Good, N (\%) & Total, N (\%) \\
\hline PICC flushing and sealing & $18.30 \pm 3.67$ & - & - & - & - \\
\hline Complication management & $26.93 \pm 7.13$ & - & - & - & - \\
\hline Overall PICC maintenance knowledge & $72.86 \pm 14.86$ & $679(16.5)$ & $2030(49.4)$ & $1401(34.1)$ & $4110(100.0)$ \\
\hline
\end{tabular}

${ }^{*}$ Grade of the total score: The total score was divided into three grades. Failed represents the score under 60 ; Passed represents the score from 60 to 79 ; Good represents a score of 80 or above.

PICC, peripherally inserted central catheter. 
Table 3 Statistics and distribution of item correct rate for different dimensions $(n=4110)$

\begin{tabular}{|c|c|c|c|c|c|c|}
\hline \multirow[b]{2}{*}{ Dimensions } & \multicolumn{4}{|c|}{ No of items by correct rate, $\mathrm{N}(\%)$} & \multirow[b]{2}{*}{ Total, N (\%) } & \multirow{2}{*}{$\begin{array}{l}\text { Correct rate } \\
(\overline{\mathrm{x}} \pm \mathrm{s})\end{array}$} \\
\hline & $<30 \%$ & $\geq 30 \%,<60 \%$ & $\geq 60 \%,<90 \%$ & $\geq 90 \%$ & & \\
\hline PICC flushing and locking & $1(8.3)$ & $1(8.3)$ & $5(41.7)$ & $5(41.7)$ & $12(100.0)$ & $0.76 \pm 0.222$ \\
\hline Complication management & $0(0.0)$ & $4(21.1)$ & $13(68.4)$ & $2(10.5)$ & $19(100.0)$ & $0.71 \pm 0.167$ \\
\hline Kruskal-Wallis $\mathrm{H}$ & 17.721 & & & & & \\
\hline$P$ value & $0.001^{*}$ & & & & & \\
\hline
\end{tabular}

${ }^{*} \mathrm{P}<0.01$.

PICC, peripherally inserted central catheter.

career and promotion as their employers could not see their scores.

The investigation was implemented by the liaisons and the head nurses who supported our study. First, we sent the electronic survey link to the liaisons by email and provided unified training on how to fill out the questionnaires by phone. Then the liaisons sent the link to the head nurses in their facilities through WeChat and told them how to fill it out. Finally, the head nurses sent the link to their Ward WeChat Group, organised the eligible nurses to fill out the questionnaires and aided the completion of the survey. The investigation was administered at 91 PICC maintenance sites at the same time from August 2017 and was kept open for 4 weeks. During the 4-week period, two email reminders were sent to the liaisons to encourage participation.

\section{Statistical analysis}

Descriptive statistics were used to summarise participant characteristics and the knowledge score of PICC maintenance. The Kruskal-Wallis $\mathrm{H}$ test was used to compare the correct rate in different dimensions. The knowledge score among nurses with different characteristics was compared using the Wilcoxon rank-sum test for the two-group comparison and the Kruskal-Wallis $\mathrm{H}$ test was used for multiple comparisons. The generalised linear model was used for multivariate analysis to identify the demographic factors influencing PICC maintenance knowledge, since the dependent variable (the knowledge score) was not normally distributed. The gamma distribution was selected as the distribution of the dependent variable and the logarithmic function was selected as the link function. All analyses were performed using SPSS V.22.0 and a two-tailed $\mathrm{p}<0.05$ value was considered statistically significant.

\section{Patient and public involvement \\ No patient involved}

\section{RESULTS}

\section{Participant characteristics}

In the present study, a total of 6524 nurses were eligible to fill out the questionnaires, and 4110 of these completed them with a response rate of $63.0 \%$. Among 4110 respondents, the majority were women $(98.6 \%)$. The largest age group ranged between 25 and 34 (51.6\%), followed by those aged under 25 (30.9\%). The majority (75.6\%) exhibited a junior professional title. All of them worked in secondary and above hospitals with $29.9 \%$ in county hospitals, $46.9 \%$ in municipal hospitals and $23.2 \%$ in provincial hospitals. Less than half of the subjects $(44.6 \%)$ reported having received PICC maintenance training previously (table 1 ).

\section{Knowledge of PICC maintenance}

The mean score of PICC maintenance among 4110 nurses was $72.86 \pm 14.86$. According to the scoring system, $83.5 \%$ of them passed the exam with a score of 60 or above and $34.1 \%$ of them exhibited a good grade with a score of 80 or above. Table 2 indicated the scores of each dimension and the overall questionnaire and the distribution of the total score.

\section{The correct rate in different dimensions}

For dimension 'PICC flushing and locking', among 12 items, two items $(16.6 \%)$ were answered with a correct rate below $60 \%$. The number of items with the correct rate below $60 \%$ for dimension 'Replacement of dressings and needle free connectors' and 'Complication management' was 5 out of $8(62.5 \%)$ and 4 out of $19(21.1 \%)$, respectively. While for dimension 'Health education' and 'Catheter removal', all items were answered with a correct rate of $60 \%$ or above (table 3 ). The difference in the correct rate among different dimensions was statistically significant $(\mathrm{H}=17.721, \mathrm{p}<0.01)$.

\section{Comparison of knowledge score among nurses with different} characteristics

As shown in table 4, statistical differences were found in PICC maintenance knowledge score among nurses 


\begin{tabular}{|c|c|c|c|}
\hline Characteristics & Score $(\bar{x} \pm s)$ & $\mathbf{Z} / \mathbf{H}$ & P value \\
\hline Gender & & $-3.280 \dagger$ & $0.001^{*}$ \\
\hline Male & $64.55 \pm 17.84$ & & \\
\hline Female & $72.98 \pm 14.78$ & & \\
\hline Age, years & & 314.543‡ & $\mathrm{p}<0.001$ \\
\hline$<25$ & $66.53 \pm 16.99$ & & \\
\hline $25-34$ & $74.98 \pm 12.86$ & & \\
\hline $35-44$ & $78.03 \pm 12.39$ & & \\
\hline$\geq 45$ & $76.29 \pm 13.33$ & & \\
\hline Professional title & & $357.421 \neq$ & $p<0.001$ \\
\hline Nurse & $67.42 \pm 16.61$ & & \\
\hline Senior nurse & $74.70 \pm 12.84$ & & \\
\hline Supervisor nurse & $78.27 \pm 11.89$ & & \\
\hline Cochief nurse & $79.40 \pm 11.54$ & & \\
\hline Education level & & $204.807 \ddagger$ & $\mathrm{p}<0.001$ \\
\hline $\begin{array}{l}\text { Technical } \\
\text { secondary school }\end{array}$ & $65.55 \pm 16.84$ & & \\
\hline Junior college & $69.35 \pm 16.58$ & & \\
\hline Undergraduate & $76.15 \pm 12.19$ & & \\
\hline Postgraduate & $74.00 \pm 14.15$ & & \\
\hline Work setting & & $79.721 \ddagger$ & $p<0.001$ \\
\hline County hospital & $70.33 \pm 14.51$ & & \\
\hline Municipal hospital & $74.28 \pm 14.72$ & & \\
\hline Provincial hospital & $73.24 \pm 15.19$ & & \\
\hline \multicolumn{2}{|c|}{$\begin{array}{l}\text { Whether PICC maintenance training } \\
\text { was obtained before? }\end{array}$} & $-17.850 \dagger$ & $p<0.001$ \\
\hline No & $69.41 \pm 14.84$ & & \\
\hline Yes & $77.14 \pm 13.73$ & & \\
\hline
\end{tabular}

${ }^{*} \mathrm{P}<0.01$.

$\dagger Z$ value.

$\ddagger \mathrm{H}$ value.

PICC, peripherally inserted central catheter;

with different gender, age, professional title, education level and work setting (all $\mathrm{p}<0.01)$. The difference in the knowledge score between nurses who had received PICC maintenance training previously and those who had not obtained training was statistically significant $(\mathrm{p}<0.01)$. The highest score (higher level of PICC maintenance knowledge) was noted among nurses who were female, aged between 35 and 44 years old with the professional title of cochief nurse and the education level of undergraduate. In addition, the highest scores were also observed for subjects working in municipal hospitals and those who had received PICC maintenance training previously (table 4).

\section{Multivariate analysis of PICC maintenance knowledge}

Multivariate analysis showed that female nurses $(\mathrm{B}=0.082$, $\mathrm{p}<0.01)$ exhibited a higher PICC maintenance knowledge score than male nurses. A lower level of PICC maintenance knowledge was noted for nurses under 25 years of age $(\mathrm{B}=-0.054, \mathrm{p}<0.05)$, those with a professional title of nurse $(\mathrm{B}=-0.074, \mathrm{p}<0.01)$, those working in county hospitals $(\mathrm{B}=-0.028, \mathrm{p}<0.01)$ and those who had not received PICC maintenance training previously $(B=-0.075$, $\mathrm{p}<0.01)$ compared with the nurses aged 45 years old or above, those with a professional title of cochief nurse, those working in provincial hospitals and those who had received PICC maintenance training before, respectively (table 5).

\section{DISCUSSION}

The present study demonstrated that the knowledge of PICC maintenance was at a medium level among nurses in Hunan province, with a mean score of $72.86 \pm 14.86$. $83.5 \%$ of nurses passed the exam with a score of 60 or above and $34.1 \%$ of them exhibited a good grade with a score of 80 or above, which was lower than that $(88.9 \%$ and $47.2 \%$ ) previously reported by Wu et al. ${ }^{26}$ This difference may result from the difference in the demographic data of the subjects. The subjects of the present study involved nurses of grade II hospitals and grade III hospitals, while the nurses in the study by $\mathrm{Wu}$ et al were all from grade III level A hospitals. This enabled the latter to have more opportunities to learn new technologies including PICC maintenance. The gap of PICC maintenance knowledge between nurses in Hunan province and those in other regions requires the attention of nursing managers, and appropriate strategies should be taken to increase their knowledge.

The nurses' level of mastery towards different dimensions was different, with the maximum correct rate of health education (90.94 \pm 0.052$)$, followed by catheter removal $(0.81 \pm 0.117)$, PICC flushing and locking $(0.76 \pm 0.222)$ and complication management $(0.71 \pm 0.167)$. The correct rate of replacement of dressings and needle free connectors was the minimum $(0.52 \pm 0.248)$ among the five dimensions. The correct rate of health education that ranked the first may be due to its uniqueness. Unlike other dimensions, health education is usually considered the transfer of knowledge without clinical operation, which is simple and easy to master. Moreover, the nurses have to repeat the knowledge again and again when educating the patients with PICC, which will strengthen their memory of the knowledge. The dimension with the minimum correct rate was the replacement of dressings and needle free connectors, which was inconsistent with that reported by Oliveira $e t a l .^{3}$ This study reported that the criteria associated with the dressing of PICC and the change of administration sets indicated a moderate to high compliance. Sharpe $e t a l^{8}$ also reported that a majority of nurses could perform well in the PICC dressings change. The difference between our findings and those of others is probably attributed to the simple way of the replacement of dressings and needle free connectors, a way that the nurses often operate according to 
Table 5 Generalised linear model of the factors influencing PICC maintenance knowledge

\begin{tabular}{|c|c|c|c|c|c|c|}
\hline \multirow[b]{2}{*}{ Factors } & \multirow[b]{2}{*}{ B } & \multirow[b]{2}{*}{ SE } & \multicolumn{2}{|c|}{ 95\% Wald Cl } & \multirow[b]{2}{*}{ Wald $\chi^{2}$} & \multirow[b]{2}{*}{$P$ value } \\
\hline & & & Lower & Upper & & \\
\hline \multicolumn{7}{|l|}{ Gender } \\
\hline Female & 0.082 & 0.0269 & 0.029 & 0.135 & 9.255 & $0.002^{* *}$ \\
\hline Male & Reference & & & & & \\
\hline \multicolumn{7}{|l|}{ Age, years } \\
\hline 25 & -0.054 & 0.0235 & -0.1 & -0.007 & 5.186 & $0.023^{*}$ \\
\hline $25-34$ & -0.003 & 0.0214 & -0.045 & 0.039 & 0.014 & 0.906 \\
\hline $35-44$ & 0.009 & 0.0202 & -0.03 & 0.049 & 0.212 & 0.645 \\
\hline$\geq 45$ & Reference & & & & & \\
\hline \multicolumn{7}{|l|}{ Professional title } \\
\hline Nurse & -0.074 & 0.0231 & -0.119 & -0.029 & 10.281 & $0.001^{* *}$ \\
\hline Senior nurse & -0.035 & 0.0208 & -0.076 & 0.006 & 2.813 & 0.094 \\
\hline Supervisor nurse & -0.002 & 0.0187 & -0.039 & 0.035 & 0.013 & 0.909 \\
\hline \multicolumn{7}{|l|}{ Cochief nurse } \\
\hline \multicolumn{7}{|l|}{ Education level } \\
\hline Technical secondary school & -0.062 & 0.0504 & -0.161 & 0.036 & 1.529 & 0.216 \\
\hline Junior college & 0.009 & 0.0413 & -0.072 & 0.09 & 0.049 & 0.824 \\
\hline Undergraduate & 0.035 & 0.041 & -0.045 & 0.116 & 0.74 & 0.390 \\
\hline Postgraduate & Reference & & & & & \\
\hline \multicolumn{7}{|l|}{ Work setting } \\
\hline County hospital & -0.028 & 0.009 & -0.046 & -0.01 & 9.768 & $0.002^{\star \star}$ \\
\hline Municipal hospital & 0.013 & 0.0081 & -0.003 & 0.029 & 2.587 & 0.108 \\
\hline Provincial hospital & Reference & & & & & \\
\hline \multicolumn{7}{|c|}{$\begin{array}{l}\text { Whether PICC maintenance training was } \\
\text { obtained before? }\end{array}$} \\
\hline No & -0.075 & 0.0066 & -0.088 & -0.062 & 128.321 & $\mathrm{p}<0.001$ \\
\hline Yes & Reference & & & & & \\
\hline
\end{tabular}

The independent variables in the generalised linear model were coadded as follows: gender (female $=0$, male $=1$ ), age $(<25=1,25-34=2,35-$ $44=3, \geq 45=4$ ), professional title (nurse $=1$, senior nurse $=2$, supervisor nurse $=3$, cochief nurse $=4$ ), education level (technical secondary school=1, junior college=2, undergraduate=3, postgraduate=4), work setting (county hospital=1, municipal hospital=2, provincial hospital=3), whether PICC maintenance training was obtained before? (no=0, yes $=1$ ) ${ }^{*} \mathrm{P}<0.05,{ }^{* *} \mathrm{P}<0.01$.

PICC, peripherally inserted central catheter.

their clinical experience, neglecting the learning toward the standardised theoretical knowledge. The gap in the mastery level towards the replacement of dressings and needle free connectors between nurses in Hunan province and those in other regions (eg, Sao Paulo, Brazil $)^{3}$ is evident. Therefore, this is a barrier that needs to be taken into consideration for nursing education and training in clinical practice. Other dimensions, such as catheter removal, PICC flushing and locking, and complication management, which exhibited a medium to high level of mastery, should not be ignored during training as they also directly affect patient safety.

The factors influencing the nurses' PICC maintenance knowledge included gender, age, professional title, work setting and previous history of PICC maintenance training. Female nurses exhibited higher PICG maintenance knowledge scores than male nurses, whereas nurses under 25 years old reported a lower level of PICC maintenance knowledge compared with those aged 45 years old or above. We did not find similar findings in other studies. Since the number of male nurses responding to the questionnaire was considerably low $(1.4 \%)$, it was unnecessary to speculate the reason for this poor performance. With regard to the contribution of the parameter age, the reason for these observations may be related to the work experience. However, Chopra $e t a t^{22}$ demonstrated no statistical difference in the knowledge of vascular nurses based on years of experience, which was possibly attributed to the small sample size. Therefore, the reason for this required further exploration.

The present study demonstrated that nurses with a professional nurse title exhibited a lower PICG 
maintenance knowledge score than those with a professional cochief nurse title. This is not surprising as nurses with senior professional titles usually possess a higher number of study and communication opportunities, which enables them to acquire additional knowledge on specialised nursing. In addition, nurses with senior professional titles often undertake clinical teaching work and gain experience when teaching interns PICC maintenance.

The most significant finding of the present study was that nurses working in county hospitals reported a lower level of PICC maintenance knowledge compared with those working in provincial hospitals. This may be due to the recent introduction of PICC maintenance technology in county hospitals. In China, specialised nursing technologies, such as PICC catheterisation and maintenance, are initially carried out by provincial hospitals and are subsequently promoted to municipal hospitals. An increasing number of county hospitals have introduced PICC maintenance technology due to the increased use of PICCs in the recent years.

To the best of our knowledge, this study is the first to compare nurses' PICC maintenance knowledge among hospitals at different levels. In consideration of the gap in the PICC maintenance knowledge between county hospitals and provincial hospitals, particular attention must be made to narrow the gap and to provide consolidated nursing for patients with PICC.

The present study further showed that nurses who had not received PICC maintenance training previously reported a lower level of PICC maintenance knowledge compared with those who received previous training, indicating that training is an effective way to enhance nurses' knowledge of PICC maintenance, which was consistent with the results reported by Roslien $e t a l^{27}$ and Purran $e t$ al. $^{28}$ Through systematic and standardised PICC training ${ }^{29}$ nurses can acquire basic knowledge of vascular anatomy, ultrasound and radiographic data evaluation skills, PICC catheterisation and maintenance standard procedures, and various emergency response and complication management processes, which could effectively decrease catheter-related complications and improve patient safety.

The present study has some limitations. First, despite using a large sample size, the study design included a cross-sectional survey and was conducted using a convenience sampling method. Therefore, non-response and selection biases may be a threat to our conclusion. Second, we investigated nurses in hospitals that had introduced PICC maintenance technology. Therefore, the findings may not be representative of the knowledge level of all nurses in Hunan province but to reflect the group with a higher level of PICC maintenance knowledge. A similar survey should be designed in the future to aim at nurses in hospitals that have not introduced PICC maintenance technology. In addition, although the quality control procedure was used throughout the data collection and entry, potential information bias may have occurred in view of the survey based on self-reported data. Finally, as mentioned in the first half of the discussion, some general information that may include factors influencing the nurses' PICG maintenance knowledge were not collected, such as years of experience. This affected our conclusion to some extent.

\section{CONCLUSIONS}

In conclusion, the knowledge of PICC maintenance was at a medium level among nurses in Hunan province, China, and this was mainly influenced by their gender, age, professional title, work setting and previous history of PICC maintenance training. However, it is important to note that the theoretical knowledge of PICC maintenance, notably in the form of guidelines, is freely available to nurses and has been used to educate nurses by the intravenous infusion team of the Xiangya Hospital within the PICC maintenance network. Therefore, the PICC maintenance knowledge of the nurses at all professional levels in all work settings could be improved. To improve the nurses' PICC maintenance knowledge, certain steps must be implemented including dissemination of the PICC maintenance knowledge in multiple ways, such as courses, lectures, seminars and new media. Particular attention must be provided to populations who responded poorly in this survey, and targeted education for nurses should also be distributed based on their performance on specific dimensions, such as the replacement of dressing and needle free connectors.

In addition, since the quality of the nurses' practical performance of PICC maintenance has a more direct impact on improving PICC-related outcomes, a study that measures nurse's practical performance should be conducted in the future. It can be measured directly by observation at bedside according to the standardised PICC maintenance order sets and checklist. It can also be measured indirectly by the PICC indwelling time, the incidence of PICC-related complications and patient satisfaction. Finally, additional measurement methods could be explored in future studies.

Acknowledgements The authors would like to thank the involved liaisons and head nurses for their help in the investigation and the participating nurses for their time allocated in this study. We would also like to thank He Lian-xiang RN, Chen Wen-feng RN, Qiu Sai-nan RN, Hu Yuan-ping RN and Chen Hua RN for their help in developing the survey instrument used in this study.

Contributors BX, JZ and ST contributed to the study conception and design, and questionnaire development. BX and JZ contributed to data collection, analysis, interpretation and manuscript drafting. $\mathrm{BX}, \mathrm{JH}$ and $\mathrm{MM}$ contributed to the literature search and data collection. BX and ZG contributed to data collection and management. All authors contributed to manuscript drafting and revising and approved the final version.

Funding This study was funded by the Natural Science Foundation of Hunan Province, China (grant no:2019JJ40501), and a Project of Management in Xiangya Hospital of Central South University (grant no: 2016 GL19). It was also supported by a key laboratory of nursing science in Hunan Province, China (grant no: 2017TP1004).

Competing interests None declared.

Patient consent for publication Not required. 
Ethics approval This study was approved by the IRB of behavioural and nursing research in School of Nursing of CSU (No 2017038).

Provenance and peer review Not commissioned; externally peer reviewed.

Data availability statement Data are available on reasonable request. Extra data can be extracted by emailing the first author BX.

Open access This is an open access article distributed in accordance with the Creative Commons Attribution Non Commercial (CC BY-NC 4.0) license, which permits others to distribute, remix, adapt, build upon this work non-commercially, and license their derivative works on different terms, provided the original work is properly cited, appropriate credit is given, any changes made indicated, and the use is non-commercial. See: http://creativecommons.org/licenses/by-nc/4.0/.

ORCID iD

Binbin Xu http://orcid.org/0000-0003-3218-7423

\section{REFERENCES}

1 Chen W, He L, Yue L, et al. Spontaneous correction of misplaced peripherally inserted central catheters. Int J Cardiovasc Imaging 2018;34:1005-8.

2 Leung T-K, Lee C-M, Tai C-J, et al. A retrospective study on the long-term placement of peripherally inserted central catheters and the importance of nursing care and education. Cancer Nurs 2011;34:E25-30.

3 Oliveira LBde, Fava YR, Rodrigues ARB, et al. Management of peripherally inserted central catheter use in an intensive care unit of a teaching hospital in Brazil: a best practice implementation project. JBI Database System Rev Implement Rep 2018;16:1874-86.

4 Zhang J, Tang S, Hu C, et al. Femorally inserted central venous catheter in patients with superior vena cava obstruction: choice of the optimal exit site. J Vasc Access 2017;18:82-8.

5 Xu B, Zhang J, Tang S, et al. Comparison of two types of catheters through femoral vein catheterization in patients with lung cancer undergoing chemotherapy: a retrospective study. J Vasc Access 2018;19:651-7.

$6 \mathrm{Yu}$ X, Yue S, Wang M, et al. Risk factors related to peripherally inserted central venous catheter nonselective removal in neonates. Biomed Res Int 2018;2018:1-6.

7 Harrod M, Montoya A, Mody L, et al. Challenges for nurses caring for individuals with peripherally inserted central catheters in skilled nursing facilities. J Am Geriatr Soc 2016;64:2059-64.

8 Sharpe E, Pettit J, Ellsbury DL. A national survey of neonatal peripherally inserted central catheter (PICC) practices. Adv Neonatal Care 2013;13:55-74

9 Goossens GA, Grumiaux N, Janssens C, et al. SecurAstaP trial: securement with SecurAcath versus StatLock for peripherally inserted central catheters, a randomised open trial. BMJ Open 2018;8:e016058.

10 Rickard CM, Marsh NM, Webster J, et al. Peripherally InSerted CEntral catheter dressing and securement in patients with cancer: the PISCES trial. Protocol for a $2 \times 2$ factorial, superiority randomised controlled trial. BMJ Open 2017;7:e015291.
11 Chopra V, Flanders SA, Saint S, et al. The Michigan appropriateness guide for intravenous catheters (MAGIC): results from a Multispecialty panel using the RAND/UCLA appropriateness method. Ann Intern Med 2015;163:S1-40.

12 Jumani K, Advani S, Reich NG, et al. Risk factors for peripherally inserted central venous catheter complications in children. JAMA Pediatr 2013;167:429-35.

13 Cotogni P, Pittiruti M. Focus on peripherally inserted central catheters in critically ill patients. World J Crit Care Med 2014;3:80-94.

14 Ohki Y, Maruyama K, Harigaya A, et al. Complications of peripherally inserted central venous catheter in Japanese neonatal intensive care units. Pediatr Int 2013:55:185-9.

15 Nolan ME, Yadav H, Cawcutt KA, et al. Complication rates among peripherally inserted central venous catheters and centrally inserted central catheters in the medical intensive care unit. J Crit Care 2016;31:238-42.

16 Piorkowska M, Al-Raweshidy Z, Yeong K. Improving peripherally inserted central catheter (PICC) care on a trauma and orthopaedics ward. BMJ Qual Improv Rep 2013;2:u464.w354.

17 Ullman AJ, Marsh N, Mihala G, et al. Complications of central venous access devices: a systematic review. Pediatrics 2015;136:e1331-44.

18 Chopra V, Anand S, Krein SL, et al. Bloodstream infection, venous thrombosis, and peripherally inserted central catheters: reappraising the evidence. Am J Med 2012;125:733-41.

19 Petroulias PL. Use of electronic tablets for patient education on flushing peripherally inserted central catheters. J Infus Nurs 2017;40:298-304.

20 Moureau NL, Dawson RB. Keeping needleless connectors clean, part 1. Nursing 2010;40:18-19.

21 Chen W, Deng H, Shen L, et al. A comprehensive intervention program on the long-term placement of peripherally inserted central venous catheters. J Cancer Res Ther 2014;10:359-62.

22 Chopra V, Kuhn L, Ratz D, et al. Vascular nursing experience, practice knowledge, and beliefs: results from the Michigan PICC1 survey. J Hosp Med 2016;11:269-75.

23 Infusion Nurses Society. Infusion therapy standards of practice. J Infus Nurs 2016;51:41-122.

24 WS/T 433-2013, Nursing practice standards for intravenous therapy [S]. [2013-11-14/2014-05-01]. Available: http://www.nhc.gov.cn/ ewebeditor/uploadfile/2014/12/20141212142815390.PDF

25 Moureau N, Lamperti M, Kelly LJ, et al. Evidence-based consensus on the insertion of central venous access devices: definition of minimal requirements for training. Br J Anaesth 2013;110:347-56.

26 Wu Q, Wu M, Yang P, et al. Status quo of knowledge-attitudebehavior of PICC pipeline maintenance in clinical nurses and analysis of its influencing factors. Chin Nurs Res 2016;30:938-41.

27 Roslien J, Alcock L. The effect of an educational intervention on the RN's peripherally inserted central catheters knowledge, confidence, and psychomotor skill. J Nurses Staff Dev 2009;25:E19-27.

28 Purran A, Weller G, Kerr C. Evaluation of a PICC care training programme. Nurs Stand 2016;30:45-50.

29 Zhang J, Tang S, He L, et al. [Effect of standardized PICC training and management on the clinical effect and complication of catheterization]. Zhong Nan Da Xue Xue Bao Yi Xue Ban 2014;39:638-43. 